\title{
Current status of endoscopic submucosal dissection for early gastric cancer in Korea: role and benefits
}

Sang Gyun Kim ${ }^{1,}$, Da Hyun Lyu ${ }^{2,}$, Chan Mi Park ${ }^{2}$, Na Rae Lee ${ }^{2}$, Jiyoung Kim², Youngju Cha ${ }^{2}$, and Hwoon-Yong Jung 3

\begin{abstract}
${ }^{1}$ Department of Internal Medicine and Liver Research Institute, Seoul National University College of Medicine, Seoul; ${ }^{2}$ National EvidenceBased Healthcare Collaborating Agency, Seoul; ${ }^{3}$ Department of Gastroenterology, Asan Medical Center, University of Ulsan College of Medicine, Seoul, Korea
\end{abstract}

Received: November 7, 2017

Revised : December 23, 2017

Accepted: December 31, 2017

\section{Correspondence to}

Hwoon-Yong Jung, M.D.

Department of Gastroenterology, Asan Medical Center, University of Ulsan College of Medicine, 88

Olympic-ro 43-gil, Songpa-gu,

Seoul 05505, Korea

Tel: +82-2-3010-3197

Fax: +82-2-479-0824

E-mail: hyjung@amc.seoul.kr

*These authors contributed equally to this work.
Background/Aims: This study was aimed to investigate the current clinical status of endoscopic submucosal dissection (ESD) for early gastric cancer (EGC) in Korea based on a National Health Insurance (NHI) database between 2011 and 2014.

Methods: The claims data of ESD for EGC in Korean NHI were reviewed using material codes of Health Insurance Review and Assessment Service between November 2011 and December 2014. The current clinical status was analyzed in terms of treatment pattern, in-hospital length of stay (LOS), total medical costs, and en bloc resection rate according to the hospital type.

Results: A total of 23,828 cases of ESD for EGC were evaluated. ESD was performed in $67.4 \%$ of cases in tertiary care hospitals, $31.8 \%$ in general hospitals, and $0.8 \%$ in hospitals, respectively. The median LOS was 5 days, and total median medical costs was approximately 1,300 US dollars. En bloc resection rate was 99\%; $8.5 \%$ of cases underwent additional treatment within 90 days ESD, and $5.5 \%$ in 91 to 365 days after ESD. The clinical status was not significantly different according to the year and hospital type.

Conclusions: A majority of ESD for EGC were performed in tertiary care hospitals in Korea. The clinical status showed excellent clinical outcomes and did not differ by the year and between the types of hospitals in Korea.

Keywords: Endoscopic submucosal dissection; Early gastric cancer; Clinical status

\section{INTRODUCTION}

Gastric cancer is the second most common malignancy in Korea [1]. Upper gastrointestinal endoscopy or barium radiologic study in population over the age of 40 years have been performed every 2 years from 1999 as part of the Korean National Cancer Screening Program, resulting in early detection rate of malignancy of over $70 \%$ among the patients with gastric cancer $[2,3]$. Furthermore, the introduction of the latest endoscopic ac- cessories and techniques of endoscopic treatment has enabled improvements in clinical outcomes by endoscopic resection for early gastric cancer (EGC) [4].

Endoscopic submucosal dissection (ESD) was introduced in Korea as the definitive treatment in the indicated cases of EGC in the early 2000's. Since November 2011, ESD has been covered by the National Health Insurance (NHI) for the cases that satisfy the absolute indications, and has been considered as a curative modality for EGC. The rate of ESD as the modality of treatment 
for EGC has steadily increased in Korea; therefore, a nationwide survey of the current status of ESD for EGC is indispensable in the evaluation of its validity as the curative method.

In this study, the current clinical status of ESD for EGC was investigated based on the NHI claims data in Korea. The treatment pattern, length of stay (LOS) in the hospital, total medical costs, and en bloc resection rate between 2011 and 2014 were evaluated according to the type of the hospital to investigate the current nationwide clinical outcomes of ESD for EGC.

\section{METHODS}

\section{Data source}

NHI claims data between 2011 and 2014 were obtained from the Health Insurance Review and Assessment Service (HIRA). The HIRA database contains information about health care utilization of the entire South Korean population; $97 \%$ of them were medical insurance subscribers and 3\% recipients of medical aid (MA). Thus, complete information about health insurance (HI) can be obtained from the HIRA database. Information in the HIRA database includes a unique identification number for each patient, demographic characteristics, and diagnoses according to the International Classification of Diseases, 1oth Revision (ICD-10) codes. Furthermore, the database includes inpatient, outpatient, pharmacy claims, the type of the hospital where an interventional procedure was performed, and history of procedures based on electronic data interchange code. The informed consent or review of ethical committee was waived because the data were collected except personal medical informations.

\section{Patient selection}

Patients were initially eligible for this study if they had a diagnosis of gastric cancer (ICD-10 codes: C16) and underwent ESD between November 2011 and December 2014. The indication for endoscopic resection prior to ESD for EGC was one of the absolute indications according to the guidelines of the Japanese Gastric Cancer Association that included differentiated-type adenocarcinoma without ulcerative findings, confinement of the lesion to the mucosa, and diameter $\leq 2 \mathrm{~cm}[5]$. We defined cases with ESD with co-payment coverage as an absolute indication. As HIRA began to cover gastric ESD as co-payment coverage when the procedure was performed for the cases meeting the absolute indication since November 2011, the first ESD for gastric cancer during the study period was considered as the index date. Additionally, the patients who underwent ESD for reasons beyond the absolute indication were included in the study.

\section{Study variables and clinical outcomes}

The database was analyzed for demographic characteristics of the patients, pattern of ESD procedure according to the hospital type, in-hospital LOS and total medical cost related to ESD. After exclusion of the cases which could not be classified as 'en bloc' or 'piecemeal' resection, en bloc resection rate of ESD was analyzed according to the hospital type and the year.

Patient demographics included sex, age, presence of chronic comorbidities, Charlson comorbidity index (CCI), hospital type and HI type. Age was stratified as < 20,20 to 29,30 to 39,40 to 49,50 to 59,60 to 69,70 to 79 , and $\geq 80$ years. Chronic comorbid conditions diagnosed within 1 year before ESD were also classified based on the ICD-10 codes: hypertension (I10-I15), diabetes mellitus (E10-E14), chronic obstructive pulmonary disease (J41-J44), renal failure ( $\mathrm{N}_{17-\mathrm{N} 19)}$, liver cirrhosis (K7OK74), angina (I20), and other cancers (Coo-C96, except C16). The severity of chronic comorbid conditions was assessed using CCI, a validated method widely used for recording comorbidities [6]. The CCI score was calculated for each patient, based on ICD-10 and expressed as the score of all comorbidities [6]. The types of hospitals in which ESD were performed were also analyzed. According to Korean medical law, healthcare institutions are categorized into six types: clinic, public health center, hospital, general hospital, tertiary care hospital, and long-term care facility. Of these, hospital, general hospital and tertiary care hospital were included in this study. The types of insurance in Korea includes wage-based contributory insurance (HI) and government-subsidized public assistance (MA).

In-hospital LOS was calculated as the days per each claim data related to ESD from the initial date of hospitalization through the date of discharge. The total medical costs were calculated per claim data as the sum of all 
costs for all the prescription medications and medical services (including hospitalization, office visits, laboratory tests, and others). Only direct cost for medical services covered under NHI were included in the study.

Additional endoscopic or surgical treatments following ESD were analyzed in patients without other cancers during the 12 months pre-index period who could be traced for 90 or 91 to 365 days after the first ESD between November 2011 and September 2014.

\section{Procedure definition}

ESD and other procedures in patients with EGC that was finally diagnosed by pathologic mapping following ESD were identified with the procedure codes with or without ESD material codes determined by HIRA. ESD was defined by the following procedure and material codes: QZ933, QX704, QX701, or Q7652. On the other hand, endoscopic mucosal resection (EMR) was defined by the following procedure codes without ESD material codes: QX701 or Q7652.

En bloc resection by ESD was identified if ESD codes (QZ933, QX704) were charged without EMR codes. If a case was charged with EMR codes (Q7652, QX701) and material codes of ESD simultaneously, we defined it as piecemeal resection by ESD. Some cases were classified as unknown if they were charged with ESD and EMR codes simultaneously, and did not correspond to the definition of en bloc or piecemeal resection. The cases with EMR codes were excluded from the analysis.

Total and subtotal gastrectomy were classified as open surgery (OP). Total gastrectomy was identified by the following codes: QA536, Q2533, Q2534, Q2536, or Q2537, while subtotal gastrectomy was identified by the following codes: Q0259, Q2594, Q0251, Q0252, Q0253, Q0254, Q0255, Q0256, Q0257, Q0258, or Q2598. Laparoscopic surgery (LAPA) was identified with total gastrectomy or subtotal gastrectomy code and material code of LAPA (Noo310001).

Regarding treatment status after ESD, the patients who underwent ESD or EMR without OP or LAPA after ESD were categorized as the endoscopic treatment group. If the patients underwent OP or LAPA without ESD or EMR, they were categorized as the surgical treatment group. Some patients who underwent both endoscopic and surgical treatment after ESD were categorized as endoscopic and surgical treatment group.

\section{Statistical analysis}

Summary statistics were calculated for continuous and categorical variables. Demographic information including age, sex, comorbidities, CCI, hospital type, en bloc resection rate, and treatment status after ESD were summarized by descriptive statistics such as frequency, proportion, mean, standard deviation, median, and range. All tests of significance were two-tailed, and $p$ values $<0.05$ were considered statistically significant. Statistical analyses were performed using SAS software version 9.2 (SAS Inc., Cary, NC, USA).

\section{RESULTS}

\section{Characteristics of patients}

A total of 23,828 ESDs for EGC were performed during the study period with an increasing trend according to the year; 1,931 cases in 2011 (November and December), 6,664 in $2012,7,499$ in 2013 , and 7,734 in 2014. The proportion of males was $74.2 \%$, and the mean and median ages were 64.9 and 66 years, respectively. The most prevalent ages were the $60 \mathrm{~s}(34.4 \%)$, and $70 \mathrm{os}(31.0 \%)$, and $50 \mathrm{~s}$ (22.6\%). Hypertension was observed in $22.6 \%$ and diabetes mellitus in $12.4 \%$ of the patients. The mean CCI was 1.1 and the score was o in $45.5 \%$ of the patients.

The type of medical insurance was $\mathrm{HI}$ in $96.2 \%$ and MA in $3.8 \%$ of the patients. ESD was performed in tertiary care hospital in $67.4 \%$, general hospital in $31.8 \%$, and hospital in $0.8 \%$ of the patients (Table 1 ).

\section{Pattern of ESD scale by hospital type}

In Korea, the number of tertiary care hospitals was 44 in 2011 to 2012 and 43 in 2013 to 2014, while there were 275 general hospitals in 2011, 278 in 2012, 281 in 2013, and 287 in 2014, and 1,375 hospitals in 2011, 1,421 in 2012, 1,451 in 2013, and 1,474 in 2014. ESD was performed in all tertiary care hospitals during the study period. The number of ESD procedures was less than 50 in $40.0 \%$ of all tertiary care hospitals, while it was 51 to 100 in $24.4 \%$, and 101 to 200 in $26.7 \%$ of them in 2012, and less than 50 in $31.1 \%$, 51 to 100 in $28.9 \%$, and 101 to 200 in $28.9 \%$ of them in 2014. More than 501 ESD procedures were performed in one institute in 2012 to 2013, and two institutes in 2014.

Among the general hospitals, ESD was performed in $28.0 \%$ of them in $2011,38.1 \%$ in $2012,40.9 \%$ in 2013 , and 
Table 1. Characteristics of patients

\begin{tabular}{|c|c|c|c|c|c|}
\hline Characteristic & $\begin{array}{c}\text { Total } \\
(\mathrm{n}=23,828)\end{array}$ & $\begin{array}{c}2011 \text { (Nov-Dec) } \\
(\mathrm{n}=1,931)\end{array}$ & $\begin{array}{c}2012 \\
(n=6,664) \\
\end{array}$ & $\begin{array}{c}2013 \\
(n=7,499)\end{array}$ & $\begin{array}{c}2014 \\
(n=7,734)\end{array}$ \\
\hline \multicolumn{6}{|l|}{ Sex } \\
\hline Male & $17,675(74.2)$ & $1,459(75 \cdot 6)$ & 4,933 (74.0) & $5,572(74 \cdot 3)$ & $5,711(73.8)$ \\
\hline Female & $6,153(25.8)$ & $472(24.4)$ & $1,731(26.0)$ & $1,927(25 \cdot 7)$ & $2,023(26.2)$ \\
\hline \multicolumn{6}{|l|}{ Age, $\mathrm{yr}^{\mathrm{a}}$} \\
\hline Mean \pm SD & $64.9 \pm 9.9$ & $63.8 \pm 10.3$ & $64.5 \pm 9.9$ & $65.2 \pm 9.9$ & $65.3 \pm 9.9$ \\
\hline Median (range) & $66(21-96)$ & $64(33-90)$ & $65(26-96)$ & $66(21-96)$ & $66(24-92)$ \\
\hline$<20$ & $\mathrm{O}$ & 0 & $\mathrm{O}$ & $\mathrm{o}$ & 0 \\
\hline $20-29$ & $14(0.1)$ & 0 & $5(0.1)$ & $6(0.1)$ & $3(0.0)$ \\
\hline $30-39$ & $163(0.7)$ & $21(1.1)$ & $40(0.6)$ & $62(0.8)$ & $40(0.5)$ \\
\hline $40-49$ & $1,373(5.8)$ & $143(7 \cdot 4)$ & $411(6.2)$ & $398(5 \cdot 3)$ & $421(5 \cdot 4)$ \\
\hline $50-59$ & $5,385(22.6)$ & $487(25.2)$ & $1,554(23 \cdot 3)$ & $1,593(21.2)$ & $1,751(22.6)$ \\
\hline $60-69$ & $8,194(34 \cdot 4)$ & $628(32.5)$ & $2,353(35 \cdot 3)$ & $2,646(35 \cdot 3)$ & $2,567(33.2)$ \\
\hline $70-79$ & $7,379(31.0)$ & $565(29 \cdot 3)$ & $1,989(29.8)$ & $2,359(31.5)$ & $2,466(31.9)$ \\
\hline$\geq 80$ & $1,320(5 \cdot 5)$ & $87(4 \cdot 5)$ & $312(4 \cdot 7)$ & $435(5.8)$ & $486(6.3)$ \\
\hline \multicolumn{6}{|l|}{ Comorbid conditions } \\
\hline Hypertension & $5,392(22.6)$ & $434(22.5)$ & $1,489(22.3)$ & $1,729(23.1)$ & $1,740(22.5)$ \\
\hline Diabetes mellitus & $2,959(12.4)$ & $256(13 \cdot 3)$ & $825(12.4)$ & $933(12.4)$ & $945(12.2)$ \\
\hline COPD & $626(2.6)$ & $47(2.4)$ & $204(3.1)$ & $197(2.6)$ & $178(2.3)$ \\
\hline Renal failure & $250(1.0)$ & $11(0.6)$ & $74(1.1)$ & $87(1.2)$ & $78(1.0)$ \\
\hline Liver cirrhosis & $315(1.3)$ & $24(1.2)$ & $83(1.2)$ & $101(1.3)$ & $107(1.4)$ \\
\hline Angina & $829(3.5)$ & $59(3.1)$ & $206(3.1)$ & $284(3.8)$ & $280(3.6)$ \\
\hline Other cancer & $1,127(4.7)$ & $84(4 \cdot 3)$ & $324(4.9)$ & $343(4.6)$ & $376(4.9)$ \\
\hline \multicolumn{6}{|l|}{ CCI } \\
\hline Mean \pm SD & $1.1 \pm 1.2$ & $1.3 \pm 1.2$ & $1.2 \pm 1.2$ & $1.1 \pm 1.2$ & $1.1 \pm 1.2$ \\
\hline $\mathrm{O}$ & $10,841(45 \cdot 5)$ & $732(37 \cdot 9)$ & $2,893(43 \cdot 4)$ & $3,451(46.0)$ & $3,765(48.7)$ \\
\hline 1 & $3,043(12.8)$ & $248(12.8)$ & $879(13.2)$ & $938(12.5)$ & $978(12.6)$ \\
\hline 2 & $6,707(28.1)$ & $640(33.1)$ & $1,929(28.9)$ & $2,113(28.2)$ & $2,025(26.2)$ \\
\hline 3 & $2,353(9.9)$ & $224(11.6)$ & $696(10.4)$ & $721(9.6)$ & $712(9.2)$ \\
\hline$\geq 4$ & $884(3.7)$ & $87(4 \cdot 5)$ & $267(4.0)$ & $276(3.7)$ & $254(3 \cdot 3)$ \\
\hline \multicolumn{6}{|c|}{ Health insurance type ${ }^{b}$} \\
\hline $\mathrm{HI}$ & $22,914(67 \cdot 4)$ & $1,856(96.1)$ & $6,426(96.4)$ & $7,220(96.3)$ & $7,421(95.8)$ \\
\hline MA & $914(31.8)$ & $75(3.9)$ & $238(3.6)$ & $279(3.7)$ & $322(4.2)$ \\
\hline \multicolumn{6}{|l|}{ Type of institution ${ }^{c}$} \\
\hline Tertiary hospital & $16,052(67.4)$ & $1,372(71.0)$ & $5,229(70.5)$ & $5,624(67.9)$ & $5,880(67.6)$ \\
\hline General hospital & $7,578(31.8)$ & $547(28.2)$ & $2,143(28.9)$ & $2,587(31.2)$ & $2,762(31.7)$ \\
\hline Hospital & $198(0.8)$ & $16(0.8)$ & $49(0.7)$ & $77(0.9)$ & $60(0.7)$ \\
\hline
\end{tabular}

Values are presented as number (\%).

SD, standard deviation; COPD, chronic obstructive pulmonary disease; CCI, Charlson comorbidity index; HI, health insurance; MA, medical aid.

${ }^{\mathrm{a}}$ Age at the time of endoscopic submucosal dissection.

${ }^{b_{T}}$ There are two types of health insurance in Korea: wage-based contributory insurance (HI) and government-subsidized public assistance (MA).

'Type of Institutions as defined by Health Insurance Review and Assessment Service in Korea. Hospital is an institution with over 30 beds aimed at medical care for inpatients. General hospital is an institution with over 100 beds, and tertiary hospital is a general hospital for professional medical practice at a high degree of difficulty for severe diseases. 
Table 2. Pattern of ESD according to the hospital type

\begin{tabular}{|c|c|c|c|c|c|}
\hline \multirow[b]{2}{*}{ No. of ESD } & \multicolumn{5}{|c|}{ No. of institution (\%) } \\
\hline & Total & $\begin{array}{c}2011 \\
(\text { Nov-Dec) }\end{array}$ & 2012 & 2013 & 2014 \\
\hline \multicolumn{6}{|l|}{ Tertiary hospital } \\
\hline$\leq 10$ & $15(8.4)$ & $9(20.9)$ & $1(2.2)$ & $2(4.4)$ & $3(6.7)$ \\
\hline $11-20$ & $18(10.1)$ & $15(34.9)$ & $2(4 \cdot 4)$ & 0 & $1(2.2)$ \\
\hline $21-30$ & $21(11.8)$ & $4(9 \cdot 3)$ & $8(17.8)$ & $5(11.1)$ & $4(8.9)$ \\
\hline $31-40$ & $16(9.0)$ & $8(18.6)$ & $4(8.9)$ & $2(4 \cdot 4)$ & $2(4 \cdot 4)$ \\
\hline $41-50$ & $12(6.7)$ & $1(2.3)$ & $3(6.7)$ & $4(8.9)$ & $4(8.9)$ \\
\hline $51-100$ & $38(21.3)$ & $3(7.0)$ & $11(24.4)$ & $11(24 \cdot 4)$ & $13(28.9)$ \\
\hline $101-200$ & $43(24.2)$ & $3(7.0)$ & $12(26.7)$ & $15(33 \cdot 3)$ & $13(28.9)$ \\
\hline $201-300$ & $5(2.8)$ & 0 & 0 & $3(6.7)$ & $2(4 \cdot 4)$ \\
\hline $301-400$ & $3(1.7)$ & 0 & $2(4 \cdot 4)$ & $1(2.2)$ & 0 \\
\hline $401-500$ & $3(1.7)$ & 0 & $1(2.2)$ & $1(2.2)$ & $1(2.2)$ \\
\hline $501-600$ & $4(2.2)$ & 0 & $1(2.2)$ & $1(2.2)$ & $2(4 \cdot 4)$ \\
\hline$\geq 601$ & o & o & o & o & o \\
\hline Total institution ${ }^{\mathrm{a}}$ & $178(100.0)$ & $43(100.0)$ & $45(100.0)$ & $45(100.0)$ & $45(100.0)$ \\
\hline \multicolumn{6}{|l|}{ General hospital } \\
\hline$\leq 10$ & $270(24.1)$ & $62(22.5)$ & $68(24 \cdot 5)$ & $65(23.1)$ & $75(26.1)$ \\
\hline $11-20$ & $62(5 \cdot 5)$ & $10(3.6)$ & $11(4.0)$ & $20(7.1)$ & $21(7 \cdot 3)$ \\
\hline $21-30$ & $24(2.1)$ & $1(0.4)$ & $9(3.2)$ & $6(2.1)$ & $8(2.8)$ \\
\hline $31-40$ & $17(1.5)$ & $2(0.7)$ & $5(1.8)$ & $7(2.5)$ & $3(1.0)$ \\
\hline $41-50$ & $9(0.8)$ & $1(0.4)$ & $2(0.7)$ & $3(1.1)$ & $3(1.0)$ \\
\hline $51-100$ & $28(2.5)$ & $1(0.4)$ & $8(2.9)$ & $9(3.2)$ & $10(3.5)$ \\
\hline $101-200$ & $9(0.8)$ & 0 & $1(0.4)$ & $5(1.8)$ & $3(1.0)$ \\
\hline $201-300$ & $4(0.4)$ & 0 & $2(0.7)$ & 0 & $2(0.7)$ \\
\hline $301-400$ & o & 0 & 0 & 0 & 0 \\
\hline Total institution ${ }^{\mathrm{a}}$ & $921(100.0)$ & $275(100.0)$ & $278(100.0)$ & $281(100.0)$ & $287(100.0)$ \\
\hline \multicolumn{6}{|l|}{ Hospital } \\
\hline$\leq 10$ & $56(1.0)$ & $8(0.6)$ & $15(1.1)$ & $19(1.3)$ & $14(0.9)$ \\
\hline $11-20$ & $1(0.0)$ & 0 & 0 & 0 & $1(0.1)$ \\
\hline$\geq 21$ & o & o & o & o & o \\
\hline Total institution ${ }^{\mathrm{a}}$ & $5,721(100.0)$ & $1,375(100.0)$ & $1,421(100.0)$ & 1,451 (100.0) & $1,474(100.0)$ \\
\hline
\end{tabular}

ESD, endoscopic submucosal dissection.

${ }^{\text {aT } T o t a l ~ n u m b e r ~ o f ~ i n s t i t u t i o n s ~ i n ~ e a c h ~ y e a r ~ b a s e d ~ o n ~} 2015$ health insurance key statistics by National Health Insurance Service of Korea.

43.6\% in 2014. The number of ESD procedures was less than 10 in $24.5 \%$ of all general hospitals, while it was 11 to 20 in $4.0 \%$ and 51 to 100 in $2.9 \%$ in 2012 , and less than 10 in $26.1 \%, 11$ to 20 in $7.3 \%$, and 51 to 100 in $3.5 \%$ in 2014 . Of the hospitals, ESD was performed in $0.6 \%$ of them in 2011, while it was $1.1 \%$ in $2012,1.3 \%$ in 2013 , and $1.0 \%$ in
2014, and the annual total number of cases was less than 10 in all hospitals except one (Table 2).

\section{In-hospital LOS}

The total LOS was 10,535 days in 2011 (November to December), 35,776 in 2012, 39,903 in 2013, and 40,720 in 2014, 


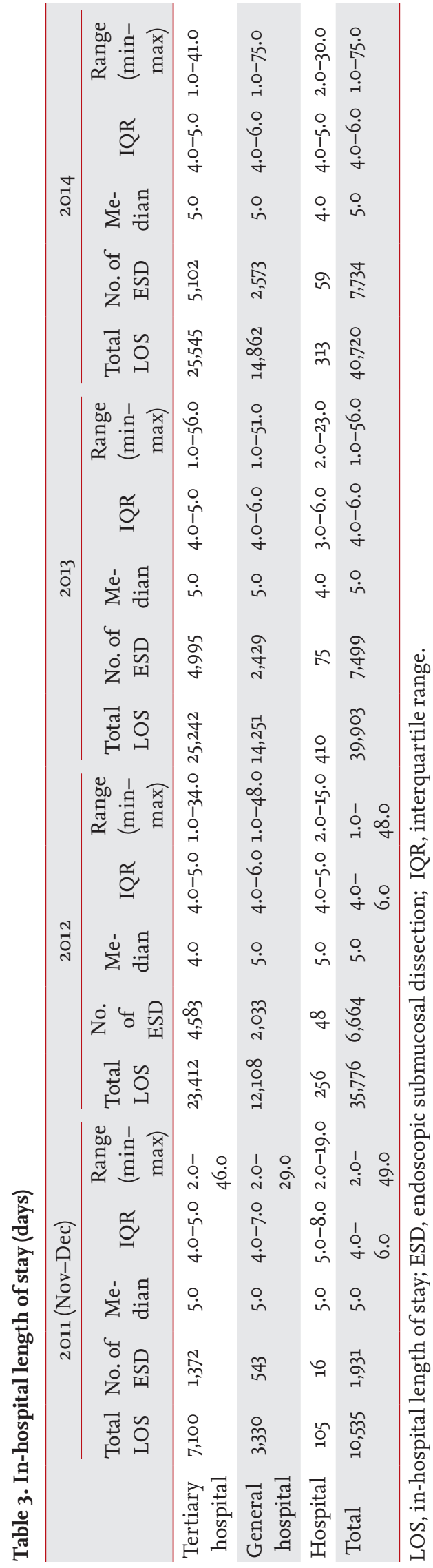

and the median LOS was 5.0 days, which did not differ according to the types of the hospital and year (Table 3 ).

\section{Total medical cost}

The costs were described in the Korean currency, won (Table 4). One US dollar (USD) was equivalent to 1,000 Korean won. The total medical cost was approximately 3.1 million USD in 2011 (November to December), 10.8 million USD in 2012, 12.2 million USD in 2013, and 13.3 million USD in 2014. The median total medical cost per patient was approximately 1,300 USD in 2011, 1,400 USD in 2012 and 2013, and 1,500 USD in 2014, which was not different between the tertiary care and general hospitals. The median total medical cost per patient in hospitals was approximately 1,100 USD in 2011, and 1,200 USD in 2012 to 2014. The annual increment in median total medical cost per patient was $2.8 \%$, which was not different from the annual increment of Korean NHI cost (2.1\%). The cost of ESD was approximately 240 USD in 2011, 250 USD in 2012 to 2013, and 400 USD in 2014.

\section{En bloc resection rate}

The en bloc resection rate was $99.0 \%$ in tertiary care hospitals, $99.1 \%$ in general hospitals, and $98.4 \%$ in hospitals, this did not differ according to the year in tertiary care and general hospitals. However, the rate in hospitals was $89.9 \%$ in 2012 , and improved up to $98.4 \%$ to $100 \%$ in 2013 to 2014 (Table 5).

\section{Endoscopic or surgical treatment status after ESD}

Additional treatment procedures were performed in $8.5 \%$ of the patients within 90 days of ESD. Of these, endoscopic treatment was performed in $1.9 \%$ and surgical treatment in $6.6 \%$ of the patients. Within 91 to 365 days of ESD, $5.5 \%$ of patients underwent additional treatments; $4.4 \%$ underwent endoscopic treatment, while $1.0 \%$ underwent surgical treatment and $0.1 \%$ underwent both (Table 6).

\section{DISCUSSION}

This is the first Korean nationwide report of the current status of ESD for EGC since the introduction of NHI coverage. The total number of cases annually of ESD for EGC were approximately 7,000 cases, which accounted 


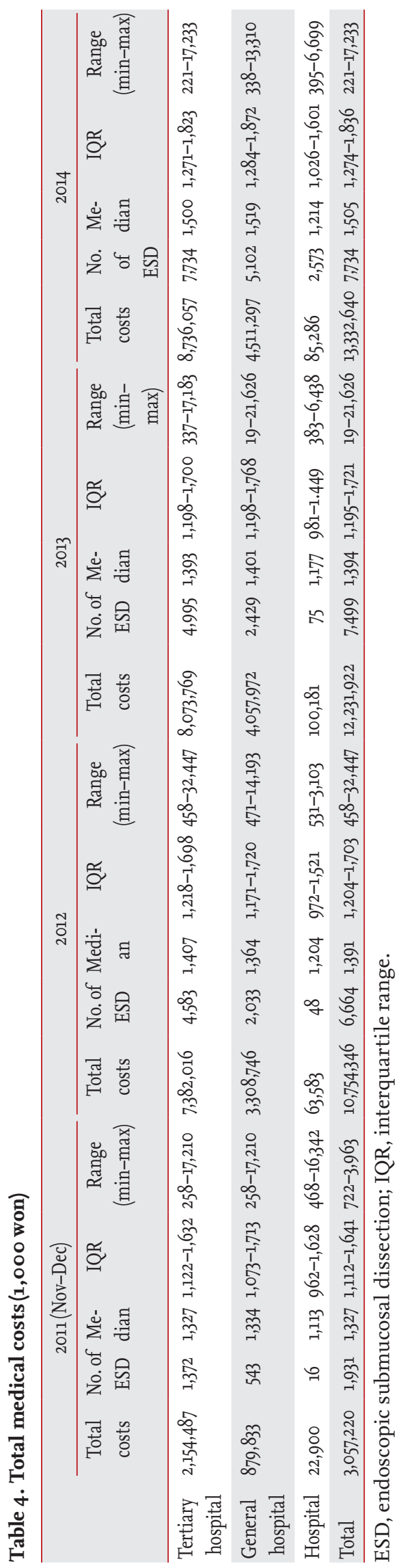

for half of total resections of EGC and one-third of total resections of all gastric cancers. Furthermore, the number of ESD have increased with years with increased early detection of cancer secondary to the expansion of the national cancer screening program.

In terms of the type of the hospital, ESD was mostly performed in tertiary care and general hospitals, and the number of ESD cases increased with years. As ESD is a more skillful endoscopic modality than conventional EMR, a back-up surgeon for procedure-related complications as well as an experienced endoscopist are needed for performing it safely. Therefore, ESD has been mostly performed in large-scaled hospitals.

The mean LOS was 5 days, which was not different according to the years or the hospital type. In most institutes, ESD was performed on the day after admission, and the patients were monitored closely for the presence of complications after initiation of diet for 2 to 3 days after ESD, which was significantly shorter than the LOS after surgical resection [7]. The reduction of LOS can reduce the total medical costs by reducing the total cost of hospital stay. A recent study highlighted the shorter LOS and lower medical costs in ESD in comparison with surgery [7].

The mean total medical cost of ESD was approximately 1,400 USD, which had increased in proportion to the increment in NHI cost according to the years. Although the total medical cost was reported to be 2,100 to 3,400 USD in a previous Korean study, the higher cost may have been because of inclusion of the cost of staging work-up as well as ESD and hospital stay [7]. The cost of ESD had increased to 400 USD since 2014, and was not different according to the hospital types. However, the total cost of ESD was significantly lower than that of surgery.

The en bloc resection rate was approximately $99.0 \%$, which was not different according to the year or hospital type. Additional procedures were performed in $8.5 \%$ of the patients; among them surgical treatment was performed in two-thirds. This result shows that the most common additional treatment was aimed at reducing the risk of residual tumor or lymph node metastasis due to incomplete resection or indications beyond the expanded criteria that were identified on final pathological mapping. However, the presence of residual tumor or lymph node metastasis could not be confirmed 
Table 5. En bloc resection rate

\begin{tabular}{|c|c|c|c|c|c|c|c|c|c|}
\hline \multirow{2}{*}{ Year } & \multicolumn{3}{|c|}{ Tertiary care hospital } & \multicolumn{3}{|c|}{ General hospital } & \multicolumn{3}{|c|}{ Hospital } \\
\hline & Total & EnR & PR & Total & EnR & PR & Total & EnR & PR \\
\hline Total $(\mathrm{n}=23, \mathrm{ol} 8)$ & 15,484 & $15,331(99.0)$ & $153(1.0)$ & 7,334 & $7,280(99.1)$ & $64(0.9)$ & 190 & $187(98.4)$ & $3(1.6)$ \\
\hline $2011($ Nov-Dec $)(n=1,885)$ & 1,340 & $1,324(98.8)$ & $16(1.2)$ & 530 & $524(98.9)$ & $6(1.1)$ & 15 & $15(100.0)$ & 0 \\
\hline $2012(\mathrm{n}=6,486)$ & 4,455 & $4,415(99.1)$ & $40(0.9)$ & 1,988 & 1,971 (99.1) & $17(0.9)$ & 43 & $43(100.0)$ & 0 \\
\hline $2013(\mathrm{n}=7,237)$ & 4,824 & $4,776(99.0)$ & $48(1.0)$ & 2,340 & $2,325(99.4)$ & $15(0.6)$ & 73 & $71(97 \cdot 3)$ & $2(2.7)$ \\
\hline $2014(\mathrm{n}=7,410)$ & 4,865 & $4,816(99.0)$ & $49(1.0)$ & 2,486 & $2,460(99.0)$ & $26(1.6)$ & 59 & $58(98.3)$ & $1(1.7)$ \\
\hline
\end{tabular}

Values are presented as number (\%).

EnR, en bloc resection; PR, piecemeal resection.

Table 6. Endoscopic or surgical treatment within 1 year after ESD

\begin{tabular}{lcc}
\hline Type of treatment & $\begin{array}{c}\text { Additional treatment within 9o days } \\
\text { after ESD }\end{array}$ & $\begin{array}{c}\text { Additional treatment within 91-365 days } \\
\text { after ESD }\end{array}$ \\
\hline Total & $20,009(100.0)$ & $14,890(100.0)$ \\
No additional treatment & $18,311(91.5)$ & $14,067(94.5)$ \\
Additional treatment & $1,698(8.5)$ & $623(5.5)$ \\
\hline Endoscopic treatment & $377(1.9)$ & $143(1.0)$ \\
\hline Surgical treatment & $1,311(6.6)$ & $20(0.1)$ \\
\hline Endoscopic \& surgical treatment & $10(0.0)$ & \\
\hline
\end{tabular}

Values are presented as number (\%).

ESD, endoscopic submucosal dissection.

in this study. Additional procedures were performed in $5.5 \%$ of the patients between 91 to 365 days after ESD; four-fifths of them underwent endoscopic treatment. This result shows that the most common additional treatment might have been performed for synchronous tumor which was found during the follow-up rather than recurrence or residue of the index tumor.

Although ESD was introduced in Korea in early 2000's, it was not covered by NHI till 2011. Therefore, ESD was performed for various indications, and the LOS, medical costs, and clinical outcomes were different among the institutes. As ESD has been widely accepted as a definitive treatment modality for EGCs with indications, the coverage by NHI for ESD in EGC in cases with absolute indications was achieved in 2011.

This study is the first nationwide report on the current status of ESD for EGC in Korea, and has the strength of inclusion of all nationwide ESD cases based on NHI data. The current status can be compared according to years and hospital types. In addition, in this study, LOS, medical costs, and clinical outcomes were evaluated according to the year and hospital types.

There are several limitations in this study. First, the individual results could not be evaluated by using the huge volume of data of NHI. Second, the real medical cost might be different from the data in NHI due to additional costs beyond the coverage. Third, the clinical efficacy of ESD was not directly compared with that of surgery. Last, as only the data within a year after ESD were evaluated, long-term clinical results were not elucidated.

In conclusion, this study has showed the nationwide current status and the benefits and roles of ESD for EGC in Korea. Further studies are needed for the evaluation of long-term efficacy of ESD for EGC with long-term follow-up. 


\section{KEY MESSAGE}

1. Endoscopic submucosal dissection (ESD) was performed in $67.4 \%$ of cases in tertiary care hospital in Korea.

2. Total median medical costs of ESD was 1,300 US dollars in Korea.

3. Clinical status of ESD was not changed by the year and among the types of hospitals in Korea.

\section{Conflict of interest}

No potential conflict of interest relevant to this article was reported.

\section{Acknowledgments}

This study was supported by research grant of the National Evidence-based Healthcare Collaborating Agency, project No. NA 16-oo1.

\section{REFERENCES}

1. Jung KW, Won YJ, Oh CM, et al. Cancer statistics in Ko- rea: incidence, mortality, survival, and prevalence in 2014. Cancer Res Treat 2017;49:292-305.

2. Suh M, Choi KS, Lee YY, Jun JK. Trends in cancer screening rates among Korean men and women: results from the Korean National Cancer Screening Survey, 2004-2012. Cancer Res Treat 2013;45:86-94.

3. Kim YG, Kong SH, Oh SY, et al. Effects of screening on gastric cancer management: comparative analysis of the results in 2006 and in 2011. J Gastric Cancer 2014;14:129134 .

4. Choi IJ, Lee NR, Kim SG, et al. Short-term outcomes of endoscopic submucosal dissection in patients with early gastric cancer: a prospective multicenter cohort study. Gut Liver 2016;10:739-748.

5. Japanese Gastric Cancer Association. Japanese gastric cancer treatment guidelines 2010 (ver. 3). Gastric Cancer 2011;14:113-123.

6. Nilssen Y, Strand TE, Wiik R, et al. Utilizing national patient-register data to control for comorbidity in prognostic studies. Clin Epidemiol 2014;6:395-404.

7. Kim Y, Kim YW, Choi IJ, et al. Cost comparison between surgical treatments and endoscopic submucosal dissection in patients with early gastric cancer in Korea. Gut Liver 2015;9:174-180. 